\title{
A NOTE ON ASSET BUBBLES IN CONTINUOUS-TIME
}

\author{
GIANLUCA CASSESE
}

\begin{abstract}
In this paper we propose a model of asset prices consistent with the no-arbitrage principle but allowing for the existence of "bubbles". The structure of bubbles is explicitely characterized and we show that, for example, they may be of either sign. Furthermore, we discuss the existence of bubbles under alternative definitions of absence of arbitrage opportunitites.

Keywords: bubbles, arbitrage, finitely additive measures, fundamental theorem of asset pricing, martingales.
\end{abstract}

JEL Classification numbers: G12, C18I.

I would like to thank Giovanni Barone-Adesi, Fabio Trojani and an anonymous referee for useful comments on an earlier draft. My gratitude also goes to Lucien Foldes for introducing me to mathematical finance. All remaining errors are my own. 


\section{INTRODUCTION}

Agents holding assets over a finite horizon earn the intermediate yields and the resale price. Viable asset prices should then depend on both these variables via a pricing map. On the other hand, the economy as a whole, may be compared to an agent that will hold the assets forever: in this perspective prices should be set to reflect the "fundamental value" of the assets, a function of dividends only. The discrepancy that may arise between these two pricing criteria is a "bubble". Bubbles arise whenever the contribution of the future resale price to the current value of the asset does not vanish no matter how far the date or remote the event of reselling the asset. As such bubbles may be viewed as a form of discontinuity of prices.

Also, viable prices do not permit arbitrage opportunities. The Fundamental Theorem of Asset Pricing establishes that the absence of arbitrage profits, conveniently defined, is equivalent to the existence of an equivalent probability measure that transforms discounted prices into martingales. When this version of the theorem is assumed, i.e. when the corresponding notion of arbitrage is adopted, bounded asset prices are just the expected, discounted value of the yields matured at all future dates. In these models pricing bubbles, which are an important issue in macroeconomics, forcefully remain outside of the picture.

In the present paper we investigate the properties of a model of financial prices in continuous time which is free of arbitrage opportunities but still does not admit equivalent martingale measures. As remarked in [9] (the first paper to adopt this setting) continuous time improves our comprehension with respect to the discrete time case. The main point is that bubbles may arise over a finite horizon, so that asset prices need not grow unboundedly.

The building block of our model is the notion of martingale density, which is considerably weaker than that of an equivalent martingale measure. Prices are turned into local martingales by virtue of a state price process which is the product of the discount factor with a positive local martingale. We shall give a few characterizations of martingale densities and describe some of their properties (in section 3). Technically speaking, unlike the case of equivalent martingale measures, the density process lacks here uniform integrability (henceforth UI). In section 2 we provide a simple example of a model with three financial assets and an American option written on two of them. We show that if the state price is a martingale density, then a mispricing arises as the price of the option remains strictly positive as its payoff converges to 0 : this discontinuity is in fact a bubble. The mispricing of exactly this option is shown to be necessary and sufficient for the existence of pricing bubbles.

This result can be put into a much more general setting. To do so we establish, in section 4, a fundamental relationship, of interest per se, between positive local martingales and finitely additive measures. One of the main attractiveness of models admitting equivalent martingale measures is that asset prices can be viewed as expected values. In our setting this conclusion still holds true although the intervening measure is not countably additive. This fact is particularly interesting and from it we derive a clear-cut decomposition of the price of an asset into its fundamental value and the bubble. We establish that pricing bubbles may have two different sources: either unbounded asset prices or lack of UI of the density process, and both components are identified explicitly. Furthermore, thanks to 
our construction, we can treat rather general price processes, thus considerably expanding our insight of this phenomenon. In our context bubbles can, for example, be either negative or positive.

A crucial step is proving that bubbles may arise in reasonable models of financial markets. This is achieved in section 5 where we show that martingale densities exclude arbitrage opportunities of a particular class. We also show a more interesting result: whenever bubbles exist, the fundamental value of an asset considerably underestimates its market value. In other words, there do exist positive contingent claims for which the fundamental value, being 0 , is not a fair price. This conclusion may be interpreted by saying that in our model bubbles do matter since they are an essential part of the fair pricing principle.

On this subject some recent work has been done by Loewenstein and Willard, mainly in [10] (but see also [9]). In these papers, in which the main focus is the notion of viability of financial prices, not addressed here, a different definition of arbitrage is proposed, based on a discussion of the role of credit constraints. Our approach differs from theirs exactly on this point, and most closely refers to [2]. Furthermore we consider general semimartingales as our model for prices rather than diffusion processes.

\section{An Example.}

This section is constructed upon an example in [8] (see p. 237 ${ }^{1}$ ). The purpose is threefold: $(i)$ to build explicitly a model in which there do not exist equivalent martingale measures; (ii) to show that the local martingale nature of the density process translates in a clear pricing anomaly; (iii) to illustrate the strict relationship between asset bubbles and the definition of an arbitrage opportunity, an issue on which we will comment extensively later on.

Consider the filtered probability space $\left(\Omega, \mathcal{F}, P ;\left(\mathcal{F}_{t}^{B}\right)_{t \in[0,1]}\right)$, where $\left(\mathcal{F}_{t}^{B}\right)_{t \in[0,1]}$ is the natural filtration of the Brownian motion $B$, define the stopping time

$$
\tau=\inf \left\{t \in[0,1]: B_{t}^{2}=(1-t)\right\}
$$

and the process $\theta_{s}=-2(1-s)^{-2} B_{s}$. The stochastic differential equation

$$
d Z_{t}=\theta_{t} Z_{t} d B_{t}
$$

admits as its unique solution the positive local martingale

$$
Z_{t}=\exp \left(-\int_{0}^{t} \frac{2 B_{s}}{(1-s)^{2}} d B_{s}-\int_{0}^{t} \frac{2 B_{s}^{2}}{(1-s)^{4}} d s\right)
$$

Since $E\left(Z_{1}\right) \leq E\left(Z_{\tau}\right)<1, Z$ is not a UI martingale. Let $\left\langle v_{n}\right\rangle$ be a sequence that localizes $Z$, $\sigma_{n}=\inf \left\{t: Z_{t} \leq 2^{-n}\right\}$ and $\tau_{n}=v_{n} \wedge \sigma_{n} \wedge 1$

Financial markets consist of three assets: two securities and the riskless asset, the price of which has been normalized to 1 . The (discounted, not necessarily positive) prices of the two securities are given respectively by

$$
R_{t}=2 \int_{0}^{t} B_{s} 1_{\{\tau \geq s\}} d s+\int_{0}^{t} 1_{\{\tau \geq s\}}(1-s) d B_{s}
$$

\footnotetext{
${ }^{1}$ The reader should consult this reference for proofs of some of the properties that we take for given.
} 
and

$$
S_{t}=\frac{E\left(Z_{1} \mid \mathcal{F}_{t}^{B}\right)}{Z_{t}}
$$

By Ito's lemma, $Z R$ is a local martingale and $Z S$ is a positive martingale: $Z$ is therefore a martingale density.

Consider an option of American style that entitles the holder to receive the riskless asset at the price of $\gamma$ units of the second security and that may be exercised at will at any random time $\tau_{n}$ : let $p_{\gamma}^{*}$ denote its price. Remark that, for a European option with maturity $\tau_{n}$, the price would necessarily be

$$
p_{\gamma, n}=E\left(\left(1-\gamma S_{\tau_{n}}\right)^{+} Z_{\tau_{n}}\right)=E\left(\left(Z_{\tau_{n}}-\gamma E\left(Z_{1} \mid \mathcal{F}_{\tau_{n}}\right)\right)^{+}\right)
$$

Clearly, $p_{\gamma}^{*} \geq p_{\gamma, n}$. It is clear that $p_{\gamma}^{*}$ is a decreasing function of $\gamma$; furthermore, $p_{\infty}^{*}(0)=0$ since the corresponding option pays nothing with probability 1 . As $\gamma$ increases, the option progressively looses the whole of its intrinsic (fundamental) value: its price may remain positive only due to the existence of a bubble, i.e. if part of its price is assigned to the event that the option will be in the money no matter the strike price. More generally:

Proposition 1. The local martingale $Z$ is a UI martingale if and only if $p_{\gamma}^{*} \downarrow 0$ as $\gamma \uparrow 1$.

Proof. If $Z$ is UI, $Z_{t}=E\left(Z_{1} \mid \mathcal{F}_{t}\right)$ (see [6], theorem I.1.42, p. 11) and $S_{\tau_{n}}=1$ : therefore $p_{\gamma}^{*}=1-\gamma$. For $b \in L^{\infty}(\Omega, \mathcal{F}, P)_{+}$and $\gamma<1$

$$
\begin{aligned}
E\left(Z_{\tau_{n}} b\right) & =E\left(\left(Z_{\tau_{n}}-\gamma E\left(Z_{1} \mid \mathcal{F}_{\tau_{n}}\right)\right)^{+} b\right)+E\left(\left(Z_{\tau_{n}} \wedge E\left(\gamma Z_{1} \mid \mathcal{F}_{\tau_{n}}\right)\right) b\right) \\
& \leq\|b\| p_{\gamma, n}(0)+E\left(\left(Z_{\tau_{n}} \wedge E\left(Z_{1} \mid \mathcal{F}_{\tau_{n}}\right)\right) b\right) \\
& \leq\|b\| p_{\gamma}^{*}(0)+E\left(E\left(Z_{1} \mid \mathcal{F}_{\tau_{n}}\right) b\right)
\end{aligned}
$$

Also $\lim _{n} E\left(E\left(Z_{1} \mid \mathcal{F}_{\tau_{n}}\right) b\right)=E\left(Z_{1} b\right)$. If $\lim _{\gamma \uparrow 1} p_{\gamma}^{*}=0$, then, by Fatou's lemma,

$$
\begin{aligned}
E\left(Z_{1} b\right) & \leq \liminf _{n} E\left(Z_{\tau_{n}} b\right) \\
& \leq \limsup _{n} E\left(Z_{\tau_{n}} b\right) \\
& \leq E\left(Z_{1} b\right)
\end{aligned}
$$

i.e. the sequence $\left\langle Z_{\tau_{n}}\right\rangle_{n \in N}$ converges weakly. This is equivalent to $\left\langle Z_{\tau_{n}}\right\rangle_{n \in \mathbb{N}}$ being UI (see [3], Theorem IV.8.9, p. 292) and the claim follows by virtue of lemma 1 below.

This proposition shows first that the lack of UI of the density process is equivalent to the mispricing of the American option, clearly a discontinuity of the pricing rule. Remark that in an economy with complete financial markets, although the option itself may not exist, it can be replicated exactly by a corresponding portfolio: this example is, so, entirely general (at least with complete markets). The proposition suggests that a test of the UI property of $Z$ could in principle be obtained by looking at the price of options out of the money and investigate whether it may be conjectured to converge to 0 with moneyness.

Second, this example is also interesting as far as arbitrage opportunities are concerned. Consider the following trading strategy: at time $t=0$ buy $a>0$ units of the second asset and sell the amount 
$a E\left(Z_{1}\right)$ of the riskless asset, holding this portfolio up to $t=1$. For any $0 \leq t \leq 1$ the value $W_{t}$ of the portfolio is $a\left(S_{t}-E\left(Z_{1}\right)\right)$. The initial cost incurred is then 0 while $W_{t}>-a$ a.s.: this is then a feasible strategy for any reasonable definition of feasibility. At maturity, $W_{1}=\left(1_{\left\{Z_{1}>0\right\}}-E\left(Z_{1}\right)\right) a$ so that $P\left(W_{1}>0\right)=P\left(Z_{1}>0\right)$, i.e. $W_{1}$ is a.s. strictly positive, unless $P\left(Z_{1}>0\right)<1$. In this example, then, a strictly positive martingale density actually implies the existence of arbitrage opportunities. This illustrates how deeply the strict positivity of the density process relates to the absence of arbitrage opportunities, the subject of the following Proposition 2.

\section{The Model}

We start with some notation. $\left(\Omega, \mathcal{F}, P ;\left\{\mathcal{F}_{t}: t \in \mathbb{R}_{+}\right\}\right)$is a filtered probability space and all processes $M=\left(M_{t}: t \in \mathbb{R}_{+}\right)$are adapted to the filtration. Put $M^{*} \triangleq \sup _{t} M_{t}$ and $M_{\infty}=\lim _{t} M_{t}$ whenever such limit exists a.s.. A UI martingale is a martingale $M$ such that the family $\left\{M_{t}: t \in \mathbb{R}_{+}\right\}$of random variables is UI. A local martingale is a process $M$ that admits a localizing sequence - i.e. a sequence $\left\langle\tau_{k}\right\rangle_{k \in N}$ of stopping times which increases to $\infty$ a.s. such that for each $k$ the stopped process $M^{k}=\left(M_{t \wedge \tau_{k}}: t \in \mathbb{R}_{+}\right)$is a UI martingale. An important result that will be repeatedly used in the sequel is Doob convergence theorem: if $M$ is a positive supermartingale then $M_{\infty}$ is well defined and a.s. finite and, consequently, $M^{*}<\infty$ a.s..

A great part of this paper hinges on the difference between local and UI martingales. A general result in the theory of stochastic processes establishes that a local martingale $M$ is a UI martingale if and only it is of class $D$, i.e. the family of random variables $\left\{M_{\tau}: \tau\right.$ a finite stopping time $\}$ is UI (see [6], proposition I.1.47, p. 11). Sufficient conditions for local martingales to be UI martingales can be found in textbooks. In our context the following, new characterization will be more useful.

Lemma 1. A local martingale $M$ is of class $D$ if and only if there exists a localizing sequence $\left\langle\tau_{n}\right\rangle_{n \in N}$ such that the collection $\left\{M_{\tau_{n}}: n \in \mathbb{N}\right\}$ is UI.

Proof. The direct implication is obvious. For the reverse one, let $\left\{M_{\tau_{n}}: n \in \mathbb{N}\right\}$ be UI for a given localizing sequence $\left\langle\tau_{n}\right\rangle$ and let $v$ be a finite stopping time. Observe that $(i)$ for any $F \in \mathcal{F}_{v}$, $\left(F \cap\left\{\tau_{n}>v\right\}\right) \in \mathcal{F}_{v} \cap \mathcal{F}_{\tau_{n}}=\mathcal{F}_{v \wedge \tau_{n}}$ and that $(i i)$ for $Y \in L_{+}^{1}(\Omega, \mathcal{F}, P)$

$$
\begin{aligned}
E\left[1_{F} E\left(Y 1_{\left\{\tau_{n}>v\right\}} \mid \mathcal{F}_{v \wedge \tau_{n}}\right)\right] & =E\left[E\left(Y \mid \mathcal{F}_{v \wedge \tau_{n}}\right) 1_{F \cap\left\{\tau_{n}>v\right\}}\right] \\
& =E\left[E\left(Y 1_{F \cap\left\{\tau_{n}>v\right\}} \mid \mathcal{F}_{v \wedge \tau_{n}}\right)\right] \\
& =E\left(Y 1_{F \cap\left\{\tau_{n}>v\right\}}\right)
\end{aligned}
$$

Since $E\left(Y 1_{\left\{\tau_{n}>v\right\}} \mid \mathcal{F}_{v \wedge \tau_{n}}\right)$ is $\mathcal{F}_{v}$ measurable, $E\left(Y 1_{\left\{\tau_{n}>v\right\}} \mid \mathcal{F}_{v \wedge \tau_{n}}\right)=E\left(Y 1_{\left\{\tau_{n}>v\right\}} \mid \mathcal{F}_{v}\right)$ i.e.

$$
E\left(Y \mid \mathcal{F}_{v \wedge \tau_{n}}\right) 1_{\left\{\tau_{n}>v\right\}}=E\left(Y \mid \mathcal{F}_{v}\right) 1_{\left\{\tau_{n}>v\right\}}
$$


But then

$$
\begin{aligned}
Z_{v} 1_{\left\{\tau_{n}>v\right\}} & =E\left(Z_{\tau_{n}} \mid \mathcal{F}_{v \wedge \tau_{n}}\right) 1_{\left\{\tau_{n}>v\right\}} \\
& =E\left(E\left(Z_{\infty} \mid \mathcal{F}_{\tau_{n}}\right) \mid \mathcal{F}_{v \wedge \tau_{n}}\right) 1_{\left\{\tau_{n}>v\right\}} \\
& =E\left(Z_{\infty} \mid \mathcal{F}_{v \wedge \tau_{n}}\right) 1_{\left\{\tau_{n}>v\right\}} \\
& =E\left(Z_{\infty} \mid \mathcal{F}_{v}\right) 1_{\left\{\tau_{n}>v\right\}}
\end{aligned}
$$

and, given that $v$ is finite a.s., $Z_{v}=E\left(Z_{\infty} \mid \mathcal{F}_{v}\right)$ follows after passing to the limit.

UI martingales are in one to one correspondence with measures, a well known fact. If $Q$ is a probability measure on the space $(\Omega, \mathcal{F})$ equivalent to $P$, then the Radon-Nikodým derivative

$$
Z_{t}=\frac{d Q_{\mathcal{F}_{t}}}{d P_{\mathcal{F}_{t}}}
$$

- where $P_{\mathcal{F}_{t}}$ and $Q_{\mathcal{F}_{t}}$ denote the restrictions of $P$ and $Q$ respectively to the $\sigma$ algebra $\mathcal{F}_{t}$ - is a strictly positive UI martingale and $Z_{0}=1^{2}$. If, further to these properties, $Z S$ is itself a (local) martingale, then $Q$ is said to be an equivalent (local) martingale measure for $S$. Whenever $Z$ and $Z S$ are just local martingales, with $Z$ non negative and $Z_{0}=1$, then $Z$ is said to be a martingale density (see [11]). Observe that martingale densities are not associated to measures. In section 4 we shall establish that such a correspondence with measures holds for local martingales as well.

The "cum dividend", discounted price process $S$ will be modeled as a vector semimartingale. No other condition is needed right now save the following

Assumption: The price process $S$ admits a martingale density $Z$.

The economic meaning of this assumption will emerge from the rest of the paper, but one issue needs clarification. In fact, it has been conjectured (in [1]) that the existence of a martingale density implies that the price process is a special semimartingales. This turns out to be incorrect.

Lemma 2. Let $S=M+V$, be a vector semimartingale, with $V$ a process of finite variation and $M$ a local martingale and let $Z$ be a positive local martingale, $Z_{0}=1$. Let $\tilde{V}=\int 1_{\left\{Z_{t->0\}}\right.} d V_{t}$, $\mathcal{L}(Z)=\int 1_{\{Z>0\}} Z^{-1} d Z$ and denote by $A^{p}$ the predictable compensator of a process of integrable variation. Then the following conditions are equivalent to $Z$ being a martingale density for $S$.

(1) $(\tilde{V}+[\mathcal{L}(Z), S])^{p}=0$;

(2) $\left\{\begin{array}{l}\tilde{V}^{c}+\left\langle\mathcal{L}(Z), M^{c}\right\rangle=0 \\ \left(\tilde{V}^{d}+\left[\mathcal{L}(Z), S^{d}\right]\right)^{p}=0\end{array}\right.$

Proof. As a consequence of the integration by parts formula we have

$$
\tilde{V}+[\mathcal{L}(Z), S]=\int \frac{1_{\left\{Z_{t-}>0\right\}}}{Z_{-}} d(S Z)-\int 1_{\left\{Z_{t-}>0\right\}} d M-\int S_{-} d \mathcal{L}(Z)
$$

\footnotetext{
${ }^{2}$ If $Z$ is only positive, then $Q$ will be absolutely continuous with respect to $P$.
} 
The left hand part is of finite variation; the right hand side is a local martingale if and only if $Z$ is a martingale density. Therefore the latter will be the case if and only if $\tilde{V}+[\mathcal{L}(Z), S]$ is a local martingale of (finite and hence of) integrable variation, a condition equivalent to (1). (1) $\rightarrow(2)$ $\tilde{V}+[\mathcal{L}(Z), M]$ being a local martingale of finite variation, its continuous part, $\tilde{V}^{c}+\left\langle\mathcal{L}(Z)^{c}, M^{c}\right\rangle$, must vanish so $0=\left(\tilde{V}^{d}+\left[\mathcal{L}(Z), M^{d}\right]\right)^{p}$, i.e. (2) follows. $(2) \rightarrow(1)$ Obvious.

$\Theta$ is the set of admissible trading strategies so that $\int \theta d S$ is well defined if $\theta \in \Theta$ then. The process $W=\left(W_{t}: t \in \mathbb{R}_{+}\right)$indicates the agent's wealth across time: therefore $W_{t}=\theta_{t} \cdot S_{t}$ for some $\theta \in \Theta$. As customary, the wealth of agents must satisfy $W_{0} \geq 0$ and the budget constraint

$$
W_{t} \leq W_{r}+\int_{r}^{t} \theta_{u} d S_{u}
$$

A negative value of $W$ represents the investor's need for funding and is subject to some restrictions in order to rule out pathological situations, like "doubling strategies", that arise when investors have unbounded access to credit. Credit constraints take the general form $\int_{0}^{t} \theta d S \geq-a \forall t$ a.s. and we define

$$
\Theta_{a}=\left\{\theta \in \Theta: \int_{0}^{t} \theta d S \geq-a\right\}
$$

The choice of how to model the bound $a$ turns out to be crucial (see [5] for a short description of alternatives). The main issue is whether the bound is fixed or it is just constrained to belong to some space $X$. In the first case borrowing constraints are exogenous and each agent has a fixed maximum amount of credit with which he is born. The only trading strategies then that can be implemented on an unbounded scale - and therefore discard the existence of equilibrium if arbitrage opportunities are available - are those in $\Theta_{a}$ with $a=0$.

In this paper we take the view that credit constraints should in principle be considered endogenous (even if we do not treat equilibrium here) and as such an increasing function of the agents ability to repay their creditors, to which potential gains from trade contribute ${ }^{3}$. If a true arbitrage opportunity exists on the market for investors with an arbitrary but limited endowment of wealth, then fund raising should become potentially unrestricted. Although most of the conclusions of the following sections hold true for different specifications of credit constraints, whenever necessary we will require $a \in L^{\infty}$, as in [2]. It is well known that credit constraints are a source of price bubbles in equilibrium models (see [7]): being rather general on this side makes therefore our results more robust.

Preliminary intuition on martingale densities as a model of asset bubbles can be obtained (along the lines developed by [9]) assuming, for the sake of the argument, that $S_{t} \geq 0$ a.s. ${ }^{4}$ and $S_{t}: \Omega \rightarrow \mathbb{R}$. In this case the positive local martingale $S Z$ is actually a supermartingale, (by Fatou's lemma) so

\footnotetext{
${ }^{3}$ In [9] (see the discussion on p. 23 and following), credit constraints are made to depend on future endowments, but not on portfolio future payoffs. This seems to impose too severe a restriction for financial intermediaries that would then be constrained in raising funds regardless of their expected performance.

${ }^{4}$ Recapitalization of firms under risk of default can be taken as an example of assets with not necessarily positive price.
} 
that

$$
Z_{t} S_{t} \geq E\left(Z_{\infty} S_{\infty} \mid \mathcal{F}_{t}\right)
$$

i.e. ${ }^{5}$

$$
S_{t}=\phi_{t}(S)+\beta_{t}(S)
$$

where $\phi_{t}(S)=Z_{t}^{-1} E\left(Z_{\infty} S_{\infty} \mid \mathcal{F}_{t}\right)$ represents the fundamental value of the asset, while $\beta_{t}(S)$ represents the non-negative bubble arising whenever the inequality in (3.7) is strict. Remark that if $Z$ is a martingale density for the price process then by (3.5) it also transforms the wealth process $W$ into a supermartingale, and, as long as it is constrained to be non negative, we obtain the decomposition (3.8) for wealth as well. Furthermore, if $Z_{\infty}>0$ a.s. the inequality (3.7) applied to portfolios also implies that no admissible trading strategy whose wealth process remains non negative throughout may be such that $P\left(W_{\infty}>0\right)>0$ - a situation that would clearly represent an arbitrage opportunity.

One of the main points of this paper is to investigate the structure of $\beta$ and to this end we will need some machinery. Furthermore, we want to extend the analysis of bubbles outside of the far too restrictive case of a non negative price or wealth processes: the supermartingale inequality will no longer apply.

\section{The Structure of Asset Bubbles}

In this section we shall show that, as well as positive UI martingales, positive local martingales too generate probability measures but only of finitely additive type. This measure theoretic characterization is entirely new and shall prove quite useful, shedding light on the structure of the pricing rule in the absence of UI. Let a localizing sequence $\left\langle\tau_{n}\right\rangle_{n \in N}$ for $Z$ be fixed throughout the rest of the paper.

As long as $\mathcal{F}_{\tau_{n}}$ measurable assets (events) are concerned, a local martingale is clearly as good a pricing and mathematical model as a UI one. In fact $\lambda(A)=E\left(Z_{\tau_{n}} 1_{A}\right)$ is a well defined (and countably additive) measure on $\left(\Omega, \mathcal{F}_{\tau_{n}}\right)$ which easily extends to $\mathcal{A}=\bigcup_{n} \mathcal{F}_{\tau_{n}}$, an algebra of subsets of $\Omega$ : the problem is rather how to extend $\lambda$ from $\mathcal{A}$ to $\mathcal{F}$. Let us remark that, for given $A \in \mathcal{F}$, when $Z$ is UI, it is equivalent to define the measure $Q$ either as $(i) Q(A)=E\left(Z_{\infty} 1_{A}\right)$ or $(i i)$ $Q(A)=\lim _{t} E\left(Z_{t} 1_{A}\right)$. In the case of $Z$ a local martingale, both definitions are unsatisfactory: the former is not an extension of $E\left(Z_{\tau_{n}} 1_{A}\right)$; the second is simply not defined ${ }^{6}$.

Let then $\Lambda$ be the linear functional on $L^{\infty}(\Omega, \mathcal{A}, P)$ defined by $\Lambda(b)=\lambda(b)$ and let $\delta$ be the functional on $L^{\infty}(\Omega, \mathcal{F}, P)$ defined by $\delta(b)=\limsup _{n} E\left(Z_{\tau_{n}} b\right)$. It is easy to see that $\delta \geq \Lambda$ on $L^{\infty}(\Omega, \mathcal{A}, P)$ and that $\delta$ is sub additive. We can then find an extension ${ }^{7}$ of $\Lambda$ to $L^{\infty}(\Omega, \mathcal{F}, P)$ (still denoted by $\Lambda$ ) such that $\Lambda(b) \leq \delta(b)$. This implies ${ }^{8}$

$$
\lim \sup _{n} E\left(Z_{\tau_{n}} b\right) \geq \Lambda(b) \geq \liminf \inf _{n} E\left(Z_{\tau_{n}} b\right)
$$

\footnotetext{
${ }^{5} S_{\infty}$ always exists on $\left\{Z_{\infty}>0\right\}$.

${ }^{6}$ Since $E\left(Z_{\tau_{n}} 1_{A}\right)$ does not converge for some $A \in \mathcal{F}$

${ }^{7}$ By the Hahn Banach theorem.

${ }^{8}$ In fact $\Lambda(b)=-\Lambda(-b) \geq-\lim \sup _{n} E\left(-Z_{\tau_{n}} b\right)=\liminf \operatorname{in}_{n} E\left(Z_{\tau_{n}} b\right)$
} 
so that $\Lambda$ is positive and bounded. By duality, $\Lambda$ is associated to a positive, finitely additive measure on $(\Omega, \mathcal{F}, P)$ which, by construction, extends $\lambda$. We shall still refer to such extension by $\lambda$. We have thus proved the existence claim in the following theorem

Theorem 1. Let $Z$ be a positive local martingale. Then $Z$ is associated to a positive, finitely additive set function $\lambda$ of bounded variation defined on $(\Omega, \mathcal{F})$ that vanishes on $P$ null sets and satisfies

$$
\lim \sup _{n} E\left(Z_{\tau_{n}} 1_{A}\right) \geq \lambda(A) \geq \liminf _{n} E\left(Z_{\tau_{n}} 1_{A}\right)
$$

for all $A \in \mathcal{F}$. Furthermore:

(1) $\lambda$ is countably additive if and only if $Z$ is a UI martingale.

(2) There exists a unique decomposition

$$
\lambda=\lambda^{c}+\lambda^{\perp}
$$

with $\lambda^{c}$ and $\lambda^{\perp}$ positive, bounded measure on $(\Omega, \mathcal{F})$ such that for any $\varepsilon>0$ and any probability measure $m$ on $(\Omega, \mathcal{F})$, there exists a set $A \in \mathcal{F}$ satisfying $\lambda^{\perp}(A)=0$ and $m\left(A^{c}\right)<\varepsilon$. Furthermore, $\lambda^{c}(A)=E\left(Z_{\infty} 1_{A}\right)$.

Proof. (claim 1). If $Z$ is UI then $\lim _{n} E\left(Z_{n} 1_{A}\right)$ exists - and coincide with $\lambda(A)$ by (4.1) - for any $A \in \mathcal{F}$ (see [3], Theorem IV.8.9, p. 292). Countable additivity then follows from Vitali-Hahn-Saks theorem (see [3], corollary III.7.3, p. 159). If $\lambda$ is countably additive, it is absolutely continuous with respect to $P$, with Radon-Nikodym derivative $U$. Since, by (4.1), $E\left(U 1_{A}\right)=\lambda(A)=E\left(Z_{\tau_{n}} 1_{A}\right)$ for any $A \in \mathcal{F}_{\tau_{n}}$, then $Z_{\tau_{n}}=E\left(U \mid \mathcal{F}_{\tau_{n}}\right)$ and $Z$ is then UI by lemma 1 .

(claim 2). Let $\lambda^{c}(A)=E\left(Z_{\infty} 1_{A}\right)$ and $\lambda^{\perp}(A)=\lim _{k} \lambda\left(A \cap\left\{\infty>Z^{*}>k\right\}\right)$. Since, $Z^{*}<\infty$ a.s. and $\lim _{n} E\left(Z_{\tau_{n}} 1_{A \cap\left\{Z^{*}<k\right\}}\right)=E\left(Z_{\infty} 1_{A \cap\left\{Z^{*}<k\right\}}\right)$ for each $A \in \mathcal{F}$ (by bounded convergence), (4.1) implies $\lambda\left(A \cap\left\{Z^{*}<k\right\}\right)=\lambda^{c}\left(A \cap\left\{Z^{*}<k\right\}\right)$. Then

$$
\begin{aligned}
0 & \leq \lambda(A)-\lambda^{c}(A) \\
& =\lim _{k} \lambda\left(A \cap\left\{\infty>Z^{*}>k\right\}\right) \\
& =\lambda^{\perp}(A)
\end{aligned}
$$

If $m$ is any probability measure, $\lambda^{\perp}\left(\left\{\infty>Z^{*}>k\right\}^{c}\right)=0$ while $m\left(\infty>Z^{*}>k\right)$ can be made arbitrary small by choosing $k$ sufficiently large. The decomposition $\lambda=\lambda^{c}+\lambda^{\perp}$ satisfies the claim and, by the theorem of Yosida and Hewitt, it is unique (see [3], theorem III.7.8, p. 163).

We now move to the characterization of bubbles. Let $\left\langle\sigma_{n}\right\rangle_{n \in N}$ be a localizing sequence for $S Z$ and $v_{n}=\sigma_{n} \wedge \tau_{n} \wedge n$. By optional stopping and for $m>k>n, S_{0}=E\left(Z_{\tau_{m}} S_{v_{k}}\right)$. Then:

$$
S_{0}=\lim _{k} \lim _{m} E\left(Z_{\tau_{m}} S_{v_{k}}\right)=\lim _{k} \lambda\left(S_{v_{k}}\right)
$$

Equation (4.3) gives then a well defined pricing rule, that replaces ordinary expected values, available when equivalent martingale measures exist. Observe that the price of an asset depends on its entire path up to maturity so that pricing derivatives by their payoff at expiry is no longer possible. One is tempted to interpret equation (4.3) in terms of bounded rationality: in order to price assets investors 
focus attention on that part of the price dynamics over which they have complete knowledge and full pricing ability, i.e. the sequence $\left\langle S_{v_{k}}\right\rangle_{k \in N}$; from this restricted information set they extrapolate the asset price by extension.

Combining (4.3) and (4.2) gives the decomposition

$$
\begin{aligned}
S_{0} & =\lim _{n} \lim _{k} \lambda\left(S_{v_{k}}\right) \\
& =\lim _{b \uparrow \infty} \lim _{n} \lim _{k}\left\{\lambda^{c}\left(S_{v_{k}} \wedge b\right)+\lambda^{c}\left(\left(S_{v_{k}}-b\right)^{+}\right)+\lambda^{\perp}\left(S_{v_{k}}\right)\right\} \\
& =\lambda^{c}\left(S_{\infty}\right)+\lim _{b \uparrow \infty} \lim _{n} \lim _{k} \lambda^{c}\left(\left(S_{v_{k}}-b\right)^{+}\right)+\lim _{n} \lim _{k} \lambda^{\perp}\left(S_{v_{k}}\right)
\end{aligned}
$$

i.e.

$$
S_{0}=\phi(S)+\beta^{u}(S)+\beta^{\perp}(S)
$$

Equation (4.4) makes clear what the sources of price bubbles are. In our model bubbles may arise because prices are unbounded - this is a necessary condition for $\beta^{u}(S) \neq 0$, a finding common to most papers on this subject. If prices are bounded, though, then $\beta^{u}(S)=0$ but there could still exist price bubbles provided $\beta^{\perp}(S) \neq 0$ : this is the case when $\lambda$ is not countably additive, i.e. $Z$ not UI Observe that indeed decomposition (4.4) applies to more general contexts than non negative prices. For prices bounded from below, for example, we still have $\beta^{u}(S) \geq 0$ although $\beta^{\perp}(S)$ may take either sign. We therefore conclude that if $\lambda^{\perp}=0$ lower bounded prices admit only positive bubbles while the reverse could be true if $\lambda^{\perp} \neq 0$.

Some further insight on the role of bubbles can be obtained from (4.4). Contrary to [9], in which this condition is necessary, we have no reason to assume that $Z_{\infty}$ is strictly positive a.s. (a condition that will be important in the next section): the example in section 2 actually shows that this assumption may generate arbitrage opportunities. In all cases in which $P\left(Z_{\infty}=0\right)>0$, there are though contingent claims that have no fundamental value and, more generally, the fundamental value operator $\phi$ underestimates the value of assets in a way that may be significant. In this case, and contrary to common intuition, bubbles not only do not distort financial prices but contribute to a correct evaluation of assets. But in the next section we will show that $P\left(Z_{\infty}=0\right)>0$ is the only case in which bubbles may exist in the absence of arbitrage opportunities.

\section{5. "Do BUbBles Matter?".}

Absence of arbitrage opportunities is a key concept and we want to investigate its implications in our model. However, the definition of arbitrage is not obvious. It is well known that the most natural and economically meaningful definition (see (5.2) below) - an admissible trading strategy bearing no cost but positive profits - need not be sufficient to recover "well behaved" price functionals. The notion of free lunch is exactly the necessary mathematical reinforcement. In continuous time models, the most significant and important notion is that of No Free Lunch with Vanishing Risk (NFLVR, introduced in [2]) that we now briefly describe. Let $X^{+}=X \vee 0$ and $X^{-}=(-X)^{+}$.

Recalling (3.6), let $\mathfrak{F}_{a}=\left\{\left(\int_{0}^{t} \theta d S: t \in \mathbb{R}_{+}\right): \theta \in \Theta_{a}\right\}$. Clearly, if $Z S$ is a local martingale and $f \in \mathfrak{F}_{a}$, then $Z f$ is a local martingale as well. For $f \in \mathfrak{F}_{a}$ define $\mathcal{S}_{f}$ to be the set of localizing sequences 
$\left\langle v_{r}\right\rangle_{r \in \mathbb{N}}$ for $f Z$ and $Z$. Define then

$$
\mathbb{K}_{a} \triangleq\left\{F \in \lambda^{0}(\Omega, \mathcal{F}, P): F=f_{\infty}, f \in \mathfrak{F}_{a}\right\}
$$

Let also $\mathbb{K} \triangleq \bigcup_{a>0} \mathbb{K}_{a}, \mathbb{C}_{a} \triangleq\left(\mathbb{K}_{a}-\lambda_{+}^{0}\right) \cap L^{\infty}, \mathbb{C} \triangleq \bigcup_{a>0} \mathbb{C}_{a}$ and let $\overline{\mathbb{C}}$ be the closure of $\mathbb{C}$ in $L^{\infty}(\Omega, \mathcal{F}, P)$.

$(N F L V R)$ is defined by the condition :

$$
\overline{\mathbb{C}} \cap L_{+}^{\infty}=\{0\}
$$

The economic meaning is more clear than the formal definition. (5.1) is violated if there exists a sequence of trading strategies such that the maximum amount of losses implied by such startegies converges to 0 uniformly, while the profits remain positive throughout ${ }^{9}$. Essentially, an agent exploiting a free lunch may pursue a positive profit while making losses small at will. This quite artificial concept is fully justified by its important consequences

Lemma 3 (Delbaen and Schachermayer 94). Let $S$ be a (locally) bounded semimartingale. NFLVR is equivalent to the existence of an equivalent (local) martingale measure $Q$.

Absence of arbitrage opportunities $(N A)$ is defined as:

$$
\mathbb{K}_{a} \cap L_{+}^{0}=\{0\}
$$

In words, an arbitrage opportunity exists if there is a trading strategy $\theta \in \Theta_{a}$ which eventually bears strictly positive net returns. Contrary to what might seem at a first glance, the definition of $N A$ does not depend on the choice of the real number $a$, provided $a>0^{10}$. In [10] and [9] (NA) is restricted to the case $a=0: Z_{\infty}>0$ a.s. is then sufficient to exclude arbitrage opportunities.

Define also

$$
\mathbb{K}_{a}^{\lambda} \triangleq\left\{F \in \mathbb{K}_{a}: F=f_{\infty} \text { and } \lim _{r} \lambda\left(\left|f_{v_{r}}^{-}-F^{-}\right|\right)=0,\left\langle v_{r}\right\rangle_{r \in \mathbb{N}} \in \mathcal{S}_{f}, f \in \mathfrak{F}_{a}\right\}
$$

and the following condition $(N A L)$ :

$$
\mathbb{K}_{a}^{\lambda} \cap L_{+}^{0}=\{0\}
$$

$\mathbb{K}_{a}^{\lambda} \subset \mathbb{K}_{a}$ implies $(N F L V R) \rightarrow(N A) \rightarrow(N A L)$.

$\mathbb{K}_{a}^{\lambda}$ differs from $\mathbb{K}_{a}$ inasmuch the negative part of $f$ is also required to converge to $F^{-}$in $L^{1}(\Omega, \mathcal{F}, \lambda)$ rather than a.s. only. In case $\lambda$ where countably additive - e.g. under (NFLVR) - this difference would be immaterial ${ }^{11}$ and, as a consequence, $\mathbb{K}_{a}=\mathbb{K}_{a}^{\lambda}$ i.e. $(N A) \longleftrightarrow(N A L)$. However, if $(N F L V R)$ is not assumed, the two convergence criteria may differ substantially. Since $\lambda$ is the pricing measure and $\left\langle v_{k}\right\rangle_{k \in N} \in \mathcal{S}_{f}, \lambda\left(f_{v_{k}}^{-}\right)$is to be interpreted as the market price for hedging the risk implicit in $f$ - e.g. a margin that some market authority may find reasonable to impose on the trader. (5.3) defines then arbitrage as a situation in which the market price of intermediate losses vanishes. The circumstance

\footnotetext{
${ }^{9}$ This interpretation is not trivial and requires to show that under $(N F L V R),\left\|f_{t}^{-}\right\| \leq\left\|f_{\infty}^{-}\right\|$for $f \in \mathfrak{F}_{a}$. This proof is obtained in the proof of the following proposition.

${ }^{10}$ In fact, for $k>0, \mathbb{K}_{k a}=k \mathbb{K}_{a}$.

${ }^{11}$ In this case, convergence in $L^{1}(\Omega, \mathcal{F}, \lambda)$ of (bounded) losses is implied by a.s. convergence.
} 
that this measure may be persistently positive even when $\lim _{k} f_{v_{k}}^{-}=0$ well captures the stylized fact for which the probability distribution implicit in financial prices displays a degree of kurtosis far above the empirical distribution. This phenomenon may be interpreted as an attitude of caution on the side of the market when assessing the costs of an arbitrage strategy.

It is difficult to establish a clear priority among definitions (5.2) and (5.3). The latter, being based on a "market" mesaure of risk, should perhaps be the relevant one for operators such as rating agencies. Nevertheless, such criterion is likely to be strongly influenced by market dynamics, such as the introduction of new securities or the diffuse bearishness of markets over a given period. The former definition, risk is measured independently and, possibly, differently from the market so that this could be the criterion adopted by outside institutions, like regulators.

Surprisingly, the relationship between martingale densities and arbitrage has not been clarified yet. We can prove the following.

Proposition 2. Let $Z$ be a martingale density and $\lambda$ the finitely additive measure associated to it. $P$ vanishing on $\lambda$ null sets is equivalent to $P\left(Z_{\infty}>0\right)=1$ and has the following implications:

(1) . (NAL) holds.

(2). (NA) is equivalent to (NFLVR).

Proof. If $P\left(Z_{\infty}>0\right)=1$, then $P$ vanishes on $\lambda^{c}$ null sets and, a fortiori, on $\lambda$ null sets. By (4.1) and reverse Fatou's lemma

$$
\lambda\left(Z_{\infty}=0, Z^{*}<k\right) \leq \lim \sup _{n} E\left(Z_{\tau_{n}} 1_{\left\{Z_{\infty}=0, Z^{*}<k\right\}}\right) \leq E\left(Z_{\infty} 1_{\left\{Z_{\infty}=0, Z^{*}<k\right\}}\right)=0
$$

Then $P\left(Z_{\infty}=0\right)=\lim _{k} P\left(Z_{\infty}=0, Z^{*}<k\right)=0$. Letting $H \triangleq c \exp \left(-Z^{*}\right)$ with $c>0$, we have $H>0$ a.s.. If $F \in \mathbb{K}_{a}$ then $F \wedge H \in \mathbb{C}_{a}$ and there are $f \in \mathfrak{F}_{a}$ and $\left\langle v_{k}\right\rangle \in \mathcal{S}_{f}$ such that $\lim _{k} f_{v_{k}}=F$. Define, for brevity, $f_{k} \triangleq f_{v_{k}}$ and $F_{k} \triangleq F-f_{k}$. Then

$$
\lambda(F \wedge H) \leq \lambda\left(F_{k} \wedge H\right)+\lambda\left(f_{k}^{+}\right) \mathrm{F} \wedge H \leq\left(F_{k} \wedge H\right)+f_{k}^{+}
$$

$$
\begin{aligned}
& \leq \lambda\left(F_{k} \wedge H\right)+\lambda\left(f_{k}^{-}\right) \mathrm{L}\left(f_{k}\right) \leq 0 \quad(5.5) \\
& \leq \lim \sup _{n} E\left(Z_{\tau_{n}}\left(F_{k} \wedge H\right)\right)+\lambda\left(f_{k}^{-}\right) \text {by }(4.1)(5.6) \\
& \leq E\left(Z_{\infty}\left(F_{k} \wedge H\right)\right)+\lambda\left(f_{k}^{-}\right) \text {reverseFatou(55nic)e } \lim \sup _{k} E\left(Z_{\infty}\left(F_{k} \wedge H\right)\right)=0 \text { and } F^{+} \wedge H=
\end{aligned}
$$
$(F \wedge H)+F^{-}$, we deduce

$$
\lambda\left(F^{+} \wedge H\right) \leq \lim \sup _{k} \lambda\left(f_{k}^{-}\right)+\lambda\left(F^{-}\right)
$$

(claim 1). By assumption, $\limsup _{k} \lambda\left(f_{k}^{-}\right)=\lambda\left(F^{-}\right)$so that $\lambda\left(F^{+} \wedge H\right) \leq 2 \lambda\left(F^{-}\right): F^{-}=0$ a.s. implies then $\lambda\left(\left(F^{+} \wedge H\right)>2^{-n}\right)=0=P\left(\left(F^{+} \wedge H\right)>2^{-n}\right)$ so that $P(F>0)=0$. (NAL) holds.

(claim 2). Let $A_{k}=\left\{f_{k} \leq-\left\|f_{\infty}^{-}\right\|\right\}$. Then $\hat{f}_{t} \triangleq 1_{A_{k}} \int_{v_{k}}^{t} d f=1_{A_{k}}\left(f_{t}-f_{k}\right)$ so that $\hat{f}_{t} \geq-a$, $\hat{f}_{\infty} \geq 0$ and $P\left(\hat{f}_{\infty}>0\right) \geq P\left(A_{k}\right)$ contradicting (5.2): therefore $\sup _{k}\left\|f_{k}^{-}\right\| \leq\left\|F^{-}\right\|$. This, together with (5.8) and $\lambda^{\perp} \geq 0$, implies $\lambda^{c}\left(F^{+} \wedge H\right) \leq 2\left\|F^{-}\right\|$. Since $H$ is defined up to a positive constant, $\lambda^{c}\left(F^{+}\right) \leq 2\left\|F^{-}\right\|$and, a fortiori, $\lambda^{c}\left(G^{+}\right) \leq 2\left\|G^{-}\right\|$if $G \in \mathbb{C}$ with $G \leq F$. But then no sequence 
in $\mathbb{C}$ may converge uniformly to a strictly positive random variable, i.e. (NFLVR) holds. Obviously $(N F L V R)$ implies $(N A)$.

Combininng proposition 2.2 and lemma 3 , we obtain the conclusion that when $Z$ is strictly positive, it is actually (or may be taken to be) UI. This contrasts with the existence of price bubbles - at least for bounded asset prices. This conclusion is important because it sheds light on the role of bubbles in asset pricing. In our model, if bubbles exist then they matter since the fundamental value criterion would provide a biased pricing rule and some contingent claims could not be priced fairly but by bubbles.

\section{REFERENCES}

[1] Back Kerry, 1991, Asset Pricing for General Processes, Journal of Mathematical Economics, 20, 371-395

[2] Delbaen Freddy and Walter Schachermayer, 1994, A General Version of the Fundamental Theorem of Asset Pricing, Mathematische Annalen, 300, 463-520.

[3] Dunford Nelson T. and Jacob Schwartz, 1988, Linear Operators. Part I, (Wiley, New-York).

[4] Harrison Michael J. and Pliska Stanley, 1981, Martingales and Stochastic Integrals in the Theory of Continuous Trading, Stochastic Processes and their Applications, 11, 215-260.

[5] Huang Kevin X. D. and Jan Werner, 2000, Asset Price Bubbles in Arrow-Debreu and Sequential Equilibrium, Economic Theory, 15, 253-278.

[6] Jacod Jean and Albert N. Shiryaev, 1987, Limit Theorems for Stochastic Processes, (Springer - Verlag, New York).

[7] Kocherlakota Narayana, 1992, Bubbles and Constraints on Debt Accumulation, Journal of Economic Theory, 37, $245-256$

[8] Liptser Robert S. and Albert N. Shiryaev, 2001, Statistics of Random Processes I. General Theory, (Springer Verlag, New York).

[9] Loewenstein Mark and Gregory A. Willard, 2000, Rational Equilibrium Asset-Pricing Bubbles in Continuous Trading Models, Journal of Economic of Theory, 91, 17-58.

[10] Loewenstein Mark and Gregory A. Willard, 2000, Local Martingales, Arbitrage and Viability: Free Snacks and Cheap Thrills, Economic Theory, 15,

[11] Schweizer Martin, 1992, Martingale Densities for General Asset Prices, Journal of Mathematical Economics, 21, 363-378.

Corresponding Address: Istituto di Economia Politica, Università Comm.le "Luigi Bocconi", via U. Gobbi, 5, 20136 Milan, Italy And, University of Southern Switzerland 